\title{
Real-World Data from a Metastatic Renal Cell Carcinoma Community- Academic Registry: Comparative Outcomes of Progression Free Survival and Overall Survival
}

\author{
Sundhar Ramalingam ${ }^{\mathrm{a}, *}$, Mark Walker ${ }^{\mathrm{b}}$, Daniel J. George ${ }^{\mathrm{a}}$ and Michael R. Harrison ${ }^{\mathrm{a}}$ \\ ${ }^{a}$ Duke Cancer Institute and the Division of Medical Oncology at the Duke University \\ School of Medicine in Durham, NC, USA \\ ${ }^{\mathrm{b}}$ Concerto HealthAI
}

\begin{abstract}
.
Background: No studies have looked at comparative outcomes in the treatment of metastatic renal cell carcinoma (mRCC) between academic and community practice settings.

Methods: We created a joint academic (ACAD) and community (COMM) retrospective registry of patients treated for mRCC. This registry represents a collaboration of an academic research network (Duke Oncology Network; Durham, NC) and a community-based oncology network (ACORN Research; Memphis, TN) of multiple member practices. We compared progression free survival and overall survival between these centers. We included patients diagnosed with mRCC after January 1, 2007 and before February 7, 2011.

Results: Four hundred and fifty-five patients were captured in the registry including N=255 COMM patients and N=200 ACAD patients. Initial analysis of COMM patients showed a median PFS of 6.24 months [95\% CI, 5.4, 7.5], 3.88 months [95\% CI, 3.0, 4.8], and 3.35 months [95\% CI 2.9, 4.4] with first, second, and third line systemic therapy. ACAD patients had longer median PFS estimates of 11.3 months [95\% CI, 7.5, 13.6], 4.4 months [95\% CI, 2.7, 8.9], and 5.22 months [95\% CI, 2.7, 6.3] respectively. Median OS was 12.06 months [95 \% CI 8.7, 15.4] among COMM patients and 36.73 months [95\% CI, 26.2, 42.2) among ACAD patients. Differences persisted with inclusion of well-established prognostic models and predictive factors such as treatment exposures.

Conclusions: There may be differences between outcomes for mRCC patients in community versus academic settings; however, selection most likely plays a role and we need further studies to determine reasons for these potential disparities. A prospective metastatic renal cell carcinoma (MaRCC) registry has been accrued encompassing sixty academic and community treatment sites across the United States, with the goal of examining real-world treatment patterns and outcomes; MaRCC may shed further light on any potential outcomes differences.
\end{abstract}

Keywords: Kidney neoplasms, renal cell carcinoma, outcome assessment, healthcare disparities

\footnotetext{
*Correspondence to: Sundhar Ramalingam, 3404 Wake Forest Road, Raleigh, NC 27609-7341, USA. Tel.: +1 919245 7127; E-mail: sr20@duke.edu.
} 


\section{INTRODUCTION}

Cancers of the kidney account for over seventy thousand malignancy diagnoses and over fourteen thousand deaths in the United States yearly [1]. Patients with metastatic renal cell cancer (mRCC), the most common histology of which is the clear cell sub-type, have historically had a median survival of less than two years [2]. After a long period of time in which there were limited treatment options for this disease, the last 13 years has seen rapid improvements with the FDA approval of 12 new drugs. Today, immunotherapy approaches with immune checkpoint inhibitors represent the newest therapies we have in treating mRCC $[3,4]$. Agents targeting the vascular endothelial growth factor receptor (VEGFR) continue to be a cornerstone of therapy, and novel approaches combining immunotherapy and VEGFR inhibition are promising approaches on the forefront [5-7]. The rapidly evolving therapeutic landscape often poses a dilemma for treating providers. The approach to treatment may vary at referral academic centers versus general community practices owing to the level of experience in certain cancer types with rapidly evolving novel agents and managing idiosyncratic toxicities. Limited studies have looked at differences between practice patterns, and to the best of our knowledge, no studies have looked at comparative outcomes in academic and community practices.

\section{METHODS}

We created a joint academic (ACAD) and community (COMM) retrospective registry of patients treated for mRCC. This registry represents a collaboration of an academic research network (Duke Oncology Network; Durham, NC) and a community-based oncology network (ACORN Research; Memphis, TN) of multiple member practices (Fig. 1). The method has been previously described $[8,9]$. Both institutional boards associated with these health systems approved this study. This registry consisted of a single set of data elements, a standardized case reporting form (CRF), data dictionary and statistical analysis plan, all of which were prepared in advance of records being selected or added. Eligible patients included adults greater than or equal to 18 years of age who received some portion of their care either at Duke University Health System (ACAD) or at an ACORN Research-affiliated

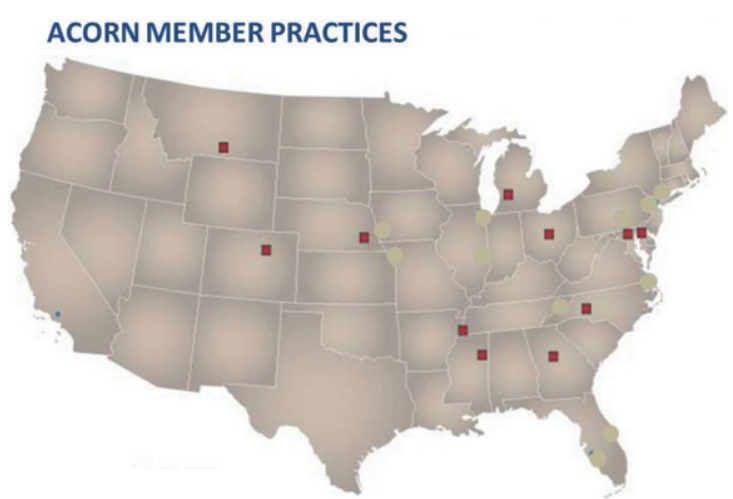

Fig. 1. Geographic locations of practices encompassing the ACORN network that contributed to this study.

community oncology site (COMM). Included were patients diagnosed with mRCC after January 1, 2007 and before February 7, 2011. January 1, 2007 was selected as a time period after which targeted therapies became widely available for clinical use. January 1,2007 was approximately one year after the approval of sorafenib and sunitinib for the treatment of mRCC.

\section{Data Sources}

Databases from both ACAD and COMM sites were queried for all eligible cases, and patients were screened approximately in the order of presentation to the respective databases. Duke University Health System routinely maintains a data warehouse including clinical, laboratory, sociodemographic, treatment and patient reported outcome data. When necessary, a review of paper charts from various oncology clinics were included. The ACORN data warehouse is a comprehensive cancer patient database including both electronic medical record and billing databases from eleven community-based oncology practices that also contains similar information. Research coordinators from each site confirmed eligibility and extracted data into a common case reporting form (CRF). Quality review was performed on completed CRFs before entry into a secure repository at which point the data was merged into a single registry.

\section{Data Analysis}

Four hundred and fifty-five records were captured in the registry including $\mathrm{N}=255 \mathrm{COMM}$ patients and $\mathrm{N}=200$ ACAD patients. Baseline demographic data was summarized using descriptive statistics. Chisquare or Fisher exact tests were used to calculate 
$p$-values for the baseline characteristics in Table 1 and 2. For the progression free survival (PFS) analysis, among the 455 patients, 172 patients were excluded due to missing data or lack of treatment (Fig. 2). Overall survival (OS) data was analyzed for 373 of the original 455 patients (82 patients had missing data) who had metastatic disease diagnosed after January 1, 2007. Baseline characteristics of these 373 patients were not believed to be significantly different compared to the original 455 patients. PFS and OS were calculated using Kaplan-Meier analysis to determine time to events. A cox regression method was used to look at the analysis of OS by source (ACAD vs COMM), as well as to adjust for covariates such as MSKCC risk, tumor grade, sites of metastasis and treatment regimens. Various treatment regimens were included in the analytical data set. These were broadly characterized as VEGFR tyrosine kinase inhibitors (TKIs) such as sunitinib and sorafenib, mTOR inhibitors such as everolimus and temsirolimus, or other medications such as bevacizumab, chemotherapy, cytokines, and non- approved combinations. The effect of commonly used therapeutic combinations during this treatment era were included, namely TKI/TKI and $\mathrm{TKI} / \mathrm{mTOR} / \mathrm{TKI}$ sequences as shown in Table 4 . The data was grouped in this way, otherwise there would have been over 150 unique sequences of therapy complicating analysis [9]. Receipt of one class of drug was considered a systemic treatment exposure, but receipt of the same drug later was not considered a new exposure. Later receipt of another drug in the same class, however, was considered a repeat exposure. Treatment exposure sequence was compared for the survival analysis based on the first three or fewer exposures. Uncommon exposure sequences of $\mathrm{N}<=2$ were grouped as "other."

\section{RESULTS}

Baseline age, gender, race, and marital status characteristics are shown (Table 1). Academic patients tended to be younger than community patients. Disease characteristics such as sites of metastasis, number of metastatic sites, histology, and performance status are also presented (Table 2). Of note, only 67 out of 200 ACAD patients were available for the first line PFS analysis compared to 216 out of 255 COMM patients. The discrepancy here may have been due to the fact that many ACAD patients may travel for consultation but continue their care at another institution whereas COMM patients may have stayed at the same practice throughout their treatment course.

Initial analysis of COMM patients showed a median PFS of 6.24 months [95\% CI, 5.4, 7.5], 3.88 months [95\% CI, 3.0, 4.8], and 3.35 months [95\% CI $2.9,4.4]$ with first, second, and third line systemic therapy. ACAD patients had longer median PFS estimates of 11.3 months [95\% CI, 7.5, 13.6], 4.4 months [95\% CI, 2.7, 8.9], and 5.22 months [95\% CI, 2.7, 6.3] respectively (Table 3 ). Overall survival is shown for 373 patients who had metastatic disease diagnosed after January 1, 2007 (Table 3). Median OS was 12.06 months [95\% CI 8.7, 15.4] among COMM patients and 36.73 months [95\% CI, 26.2, 42.2) among ACAD patients (Fig. 3b). Cox regression analysis showing the effect on OS by source (ACAD vs COMM), and other covariates including MSKCC risk class, and treatment exposure sequence by source is also presented (Table 4). Differences in OS across the total study population were seen by treatment sequence. The difference in OS persisted after including known prognostic factors, such as treatment sequence, presence of liver metastasis, and Fuhrman grade in the model.

\section{DISCUSSION}

Our results, to the best of our knowledge, are the first comparative report of outcomes between academic and community treatment centers. The results indicate differences in both progression free survival and overall survival for patients treated for $\mathrm{mRCC}$ between ACAD and COMM groups (Fig. 3A, 3B). These differences persist with inclusion of wellestablished prognostic models (MSKCC risk score, presence of liver metastasis, Fuhrman Grade) and predictive factors such as treatment exposure sequence (VEGFR TKI, mTOR, other). It should also be noted that both PFS and OS outcomes seem to be inferior with the COMM data compared to what has been reported in first-line studies of targeted therapies during this treatment time period $[10,11]$.

Limitations of the study include the retrospective nature of the analysis, which allows for selection bias, specifically the possibility of comparing inherently different patient populations. There may have been differences in unmeasured variables. Although data examined for the purpose of this analysis allowed controlling for well-established prognostic and predictive factors, information regarding other potential 
Table 1

Demographics

\begin{tabular}{lcccc}
\hline & $\begin{array}{c}\text { Community } \\
(\mathrm{N}=255)\end{array}$ & $\begin{array}{c}\text { Academic } \\
(\mathrm{N}=200)\end{array}$ & $\begin{array}{c}\text { Overall } \\
(\mathrm{N}=455)\end{array}$ & $P$-value \\
\hline Age (years) & & & & \\
$\quad$ Mean (SD) & $65.4(10.77)$ & $61.2(9.53)$ & $63.6(10.44)$ & $<0.0001$ \\
Median & 65.3 & 61.2 & 63.1 & \\
$\mathrm{n}$ & 254 & 193 & 447 & 0.549 \\
Gender, n (\%) & & & & \\
$\quad$ Male & $172(67.5 \%)$ & $128(64.6 \%)$ & $300(66.2 \%)$ & \\
Female & $83(32.5 \%)$ & $70(35.4 \%)$ & $153(33.8 \%)$ & \\
Race, n (\%) & 0 & & & \\
$\quad$ Asian & 0 & $3(1.5 \%)$ & $3(0.7 \%)$ & \\
Black or African American & $34(13.3 \%)$ & $26(13.0 \%)$ & $60(13.2 \%)$ & \\
Hispanic or Latino & 0 & $4(2.0 \%)$ & $4(0.9 \%)$ & \\
White & $181(71.0 \%)$ & $145(72.5 \%)$ & $326(71.6 \%)$ & \\
Other & $3(1.2 \%)$ & 0 & $3(0.7 \%)$ & \\
$\quad$ Not in Record & $37(14.5 \%)$ & $22(11.0 \%)$ & $59(13.0 \%)$ & \\
Marital Status Category, $\mathrm{n}(\%)$ & & & \\
$\quad$ Married/Living together & $165(64.7 \%)$ & $137(68.5 \%)$ & $302(66.4 \%)$ & \\
$\quad$ Not Married/Not Living together & $90(35.3 \%)$ & $63(31.5 \%)$ & $153(33.6 \%)$ & \\
\hline
\end{tabular}

Table 2

Disease characteristics at initial diagnosis

\begin{tabular}{|c|c|c|c|c|}
\hline & $\begin{array}{l}\text { Community } \\
(\mathrm{N}=255)\end{array}$ & $\begin{array}{l}\text { Academic } \\
(\mathrm{N}=200)\end{array}$ & $\begin{array}{c}\text { Overall } \\
(\mathrm{N}=455)\end{array}$ & $P$-value \\
\hline \multicolumn{5}{|l|}{ Metastatic Sites*, n(\%) } \\
\hline Brain & $14(5.5 \%)$ & $4(2.0 \%)$ & $18(4.0 \%)$ & 0.058 \\
\hline Lung & $128(50.2 \%)$ & $103(51.5 \%)$ & $231(50.8 \%)$ & 0.782 \\
\hline Liver & $35(13.7 \%)$ & $33(16.5 \%)$ & $68(14.9 \%)$ & 0.410 \\
\hline Bone & $99(38.8 \%)$ & $50(25.0 \%)$ & $149(32.7 \%)$ & 0.0018 \\
\hline Other & $107(42.0 \%)$ & $65(32.5 \%)$ & $172(37.8 \%)$ & 0.0389 \\
\hline Total Number of Metastatic Sites & & & & 0.0016 \\
\hline Mean (SD) & $1.6(0.9)$ & $1.4(0.67)$ & $1.5(0.84)$ & \\
\hline Median (range) & $1(1,5)$ & $1(1,4)$ & $1(1,5)$ & \\
\hline $\mathrm{n}$ & 254 & 140 & 394 & \\
\hline \multicolumn{5}{|l|}{ Histologic Type of $\mathrm{RCC}^{*}, \mathrm{n}(\%)$} \\
\hline Chromophobe & $3(1.2 \%)$ & $8(4.0 \%)$ & $11(2.4 \%)$ & 0.066 \\
\hline Clear cell & $157(61.6 \%)$ & $142(71.0 \%)$ & $299(65.7 \%)$ & 0.035 \\
\hline Collecting duct & $2(0.8 \%)$ & $2(1.0 \%)$ & $4(0.9 \%)$ & 1.0 \\
\hline Oncocytoma & $1(0.4 \%)$ & $1(0.5 \%)$ & $2(0.4 \%)$ & 1.0 \\
\hline Papillary & $11(4.3 \%)$ & $10(5.0 \%)$ & $21(4.6 \%)$ & 0.72913 \\
\hline Sarcomatoid features & $11(4.3 \%)$ & $10(5.0 \%)$ & $21(4.6 \%)$ & 0.72913 \\
\hline Unclassified & $1(0.4 \%)$ & 0 & $1(0.2 \%)$ & 1.0 \\
\hline Other & $13(5.1 \%)$ & $3(1.5 \%)$ & $16(3.5 \%)$ & 1.0 \\
\hline Unknown/not documented & $66(25.9 \%)$ & $45(22.5 \%)$ & $111(24.4 \%)$ & 0.40439 \\
\hline Composite Performance Status, n (\%) & & & & 0.4673 \\
\hline Impaired & $33(12.9 \%)$ & $21(10.5 \%)$ & $54(11.9 \%)$ & \\
\hline Not Impaired, or not present & $222(87.1 \%)$ & $179(89.5 \%)$ & $401(88.1 \%)$ & \\
\hline Fuhrman Grade, n (\%) & & & & 0.0036 \\
\hline II & $31(12.3 \%)$ & $47(25.5 \%)$ & $78(17.9 \%)$ & \\
\hline III & $60(23.8 \%)$ & $44(23.9 \%)$ & $104(23.9 \%)$ & \\
\hline IV & $29(11.5 \%)$ & $16(8.7 \%)$ & $45(10.3 \%)$ & \\
\hline Unknown/Not documented & $132(52.4 \%)$ & $77(41.8 \%)$ & $209(47.9 \%)$ & \\
\hline \multicolumn{5}{|l|}{ Surgical Procedures*, n (\%) } \\
\hline Nephrectomy & $166(65.1 \%)$ & $164(82.0 \%)$ & $330(72.5 \%)$ & 0.00006 \\
\hline Partial nephrectomy & $3(1.2 \%)$ & $5(2.5 \%)$ & $8(1.8 \%)$ & 0.475 \\
\hline Nodal Dissection & 0 & $16(8.0 \%)$ & $16(3.5 \%)$ & $<0.00001$ \\
\hline Cryotherapy/RFA & $1(0.4 \%)$ & 0 & $1(0.2 \%)$ & 1.0 \\
\hline Metastectomy & $23(9.0 \%)$ & $6(3.0 \%)$ & $29(6.4 \%)$ & 0.009 \\
\hline
\end{tabular}

*Patients may have had multiple results in these assessment categories. Overall chi square $p$ values were provided for categorical variables with mutually exclusive categories. 


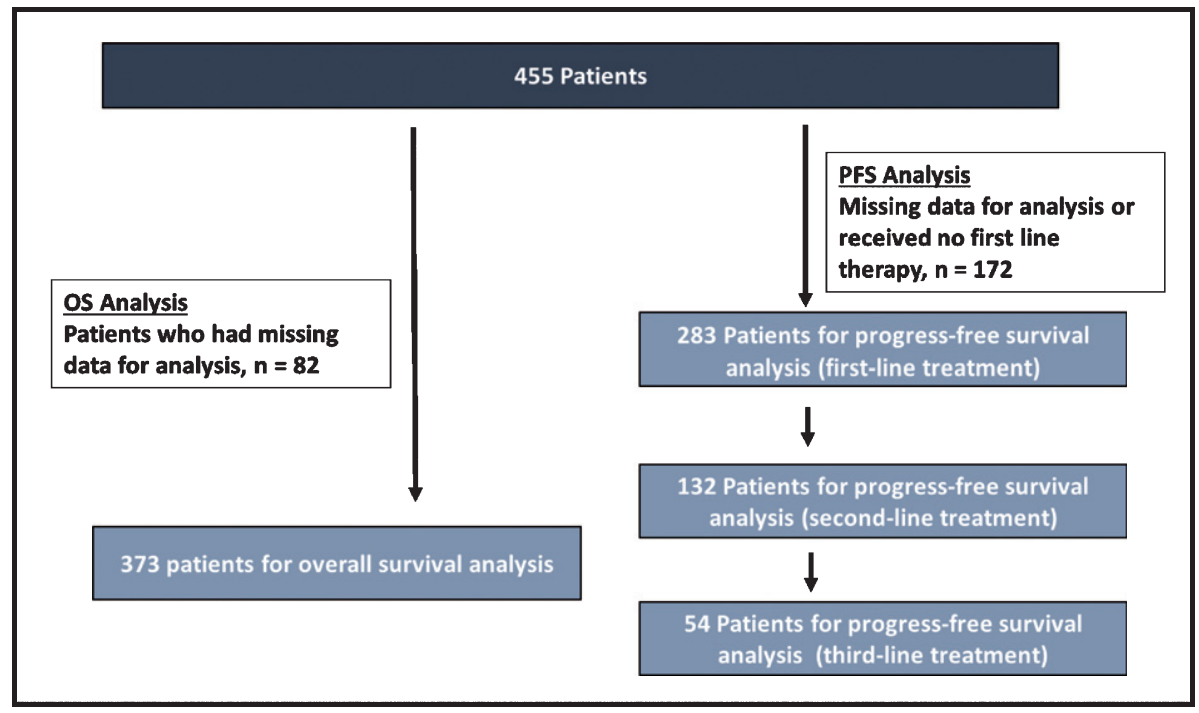

Fig. 2. Consort diagram of study cohort analyzed for progression free survival.

Table 3

Summary of Progression Free and Overall Survival

\begin{tabular}{lcc}
\hline & Community & Academic \\
\hline PFS, Therapy Line 1 & & \\
No. of Events/No. of Subjects & $187 / 216$ & $54 / 67$ \\
Mean in months (SD) & $9.35(0.62)$ & $15.37(1.7)$ \\
Median in months [95\% CI] & $6.24[5.4,7.5]$ & $11.3[7.5,13.6]$ \\
Logrank $p$-value & 0.0006 & \\
PFS, Therapy Line 2 & & \\
No. of Events/No. of Subjects & $89 / 109$ & $21 / 23$ \\
Mean in months (SD) & $5.92(0.6)$ & $9.71(2.26)$ \\
Median in months [95\% CI] & $3.88[3.0,4.6]$ & $4.40[2.7,8.9]$ \\
Logrank $p$-value & 0.2404 & \\
PFS, Therapy Line 3 & & $8 / 10$ \\
No. of Events/No. of Subjects & $38 / 44$ & $4.8(0.47)$ \\
Mean in months (SD) & $4.48(0.5)$ & $5.22[2.7,6.3]$ \\
Median in months [95\% CI] & $3.35[2.9,4.4]$ & \\
Logrank $p$-value & 0.4098 & $58 / 119$ \\
OS & & $31.16(1.85)$ \\
No. of Events/No. of Subjects & $159 / 254$ & \\
Mean in months (SD) & $17.53(1.03)$ & \\
Median in months [95\% CI] & $12.06[8.7,15.4]$ & $36.73[26.2,42.2]$ \\
Logrank $p$-value & $<0.0001$ & \\
\hline
\end{tabular}

confounding variables are lacking. Retrospective analysis of standard of care medical records may be of poor quality or with missing values. The data is also limited without further explanation as to what are the potential specific reasons for patient-related (socioeconomic status, comorbidity, literacy level, health access limitations) and provider-related (drug dosing, intensity, side effect management practices, availability of access to ancillary health services) causes for differences in outcomes. Baseline characteristics deferred including a lower median age of 61.2 years in ACAD patients versus 65.4 years in COMM patients. This may have contributed to differences is comorbidity and survival between groups. ACAD patients also had a percentage numerically lower incidence of brain metastasis $(2.0 \%$ vs $5.5 \%)$ and statistically significant more bone metastasis (38.8\% vs $25.0 \%$ ), as well as a statistically higher incidence of clear cell histology (71.0\% vs 61.6\%), nephrectomy ( 82.0 $\%$ vs. $65.1 \%)$ and nodal dissections (8\% vs. $0 \%)$. 

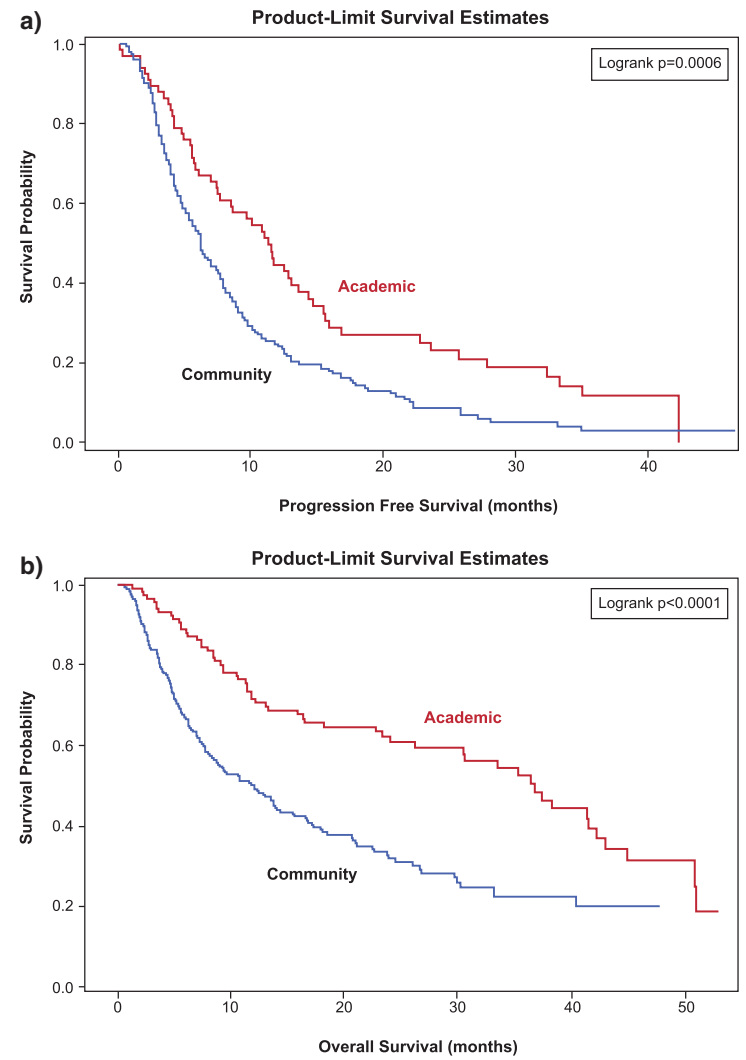

Fig. 3. Kaplan-Meier curves of (a) progression free survival (months), by first line therapy and (b) overall survival (months) for patients diagnosed with mRCC after January 1, 2007.
Metastectomy was statistically more common in the COMM population (9\% vs 3\%). Significant differences in Fuhrman grade was established with a higher incidence of grade II disease in the ACAD population and grade IV disease in the COMM population. The data presented also represents an analysis from only one academic center and one community network, so the data may not be generalizable in a broader sense.

Despite these limitations, the analysis presented does warrant further investigation into the potential reasons for disparity. One hypothesis is that there may be differences in the approach to management of adverse events, which may lead to differences in outcomes. Our data does not strongly suggest that a particular difference in treatment or therapeutic sequence accounts for the differences in PFS and OS between ACAD and COMM. However, our Cox model suggests there may be varying degrees of effect on outcomes between ACAD and COMM for particular treatments and sequences. It is possible that this could be attributable to practice differences in drug selection and therapeutic management. This data is from a treatment era prior to 2011 when targeted agents including VEGFR-TKIs and mTOR inhibitors were the mainstay of therapy, and also a time period where these drugs were just starting to become widely used. Although very efficacious medications, targeted therapies are known for their high

Table 4

Summary of Cox regression analysis of OS

\begin{tabular}{|c|c|c|c|c|c|}
\hline & Estimate & $\begin{array}{l}\text { Standard } \\
\text { Error }\end{array}$ & $\begin{array}{c}\text { Chi-Square } \\
p \text {-value }\end{array}$ & $\begin{array}{c}\text { Hazard } \\
\text { Ratio } \\
\text { (Mortality) }\end{array}$ & $\begin{array}{c}\text { 95\% Hazard } \\
\text { Ratio } \\
\text { Confidence Limits } \\
\end{array}$ \\
\hline Regimen Class Group Overall $P$-value & & & $<0.0001$ & & \\
\hline Regimen Group TKI/TKI vs. TKI & -1.55466 & 0.36218 & $<0.0001$ & 0.211 & $0.104-0.430$ \\
\hline Regimen Group TKI/mTOR/TKI vs. TKI & -1.75597 & 0.46239 & 0.0001 & 0.173 & $0.070-0.428$ \\
\hline Regimen Group mTOR vs. TKI & -0.06212 & 0.29223 & 0.8316 & 0.94 & $0.530-1.666$ \\
\hline Regimen Group Other vs. TKI & -1.32167 & 0.36256 & 0.0003 & 0.267 & $0.131-0.543$ \\
\hline Metastatic liver site Yes vs. No & 0.65654 & 0.19777 & 0.0009 & 1.928 & $1.309-2.841$ \\
\hline Fuhrman grade III/IV vs. I/II & 0.8492 & 0.27867 & 0.0023 & 2.338 & $1.354-4.037$ \\
\hline Fuhrman grade Unknown vs. I/II & 0.77359 & 0.27199 & 0.0045 & 2.168 & $1.272-3.694$ \\
\hline Fuhrman Grade Overall $P$-value & & & 0.0077 & & \\
\hline COMM vs. ACAD & 1.71089 & 0.30253 & $<0.0001$ & 5.534 & $3.059-10.012$ \\
\hline Regimen Group TKI/TKI by Source & 1.55644 & 0.66023 & 0.0184 & 4.742 & $1.300-17.296$ \\
\hline Regimen Group TKI/mTOR/TKI by Source & 2.65663 & 0.77576 & 0.0006 & 14.248 & $3.115-65.177$ \\
\hline Regimen Group mTOR by Source & 1.20036 & 0.62866 & 0.0562 & 3.321 & $0.969-11.387$ \\
\hline Regimen Group Other by Source & 1.34978 & 0.53885 & 0.0122 & 3.857 & $1.341-11.089$ \\
\hline Regimen Group by Source Overall $P$-value & & & 0.0028 & & \\
\hline MSKCC Moderate vs. High & -0.72295 & 0.22479 & 0.0013 & 0.485 & $0.312-0.754$ \\
\hline MSKCC Low vs. High & -1.82496 & 0.28922 & $<0.0001$ & 0.161 & $0.091-0.284$ \\
\hline MSKCC Overall $P$-value & & & $<0.0001$ & & \\
\hline
\end{tabular}

COX model using backward selection method with significant level 0.05 . Several sequence groups (TKI/TKI/mTOR, TKI/mTOR, mTOR/TKI) were excluded due to too few cases from Duke. 
rate of toxicities, and it is speculative but possible that differences in experience of managing toxicities between ACAD and COMM centers could have led to less dose interruptions and discontinuations at ACAD centers potentially impacting outcomes. Retrospective registry data have also recently indicated improved outcomes for patients with mRCC treated at higher volume facilities [12]. VEGFRTKIs are known to cause a number of adverse events including hypertension, gastritis, nausea, diarrhea, hand-foot syndrome, rash, endocrinopathies, nephrotoxicity, and hepatotoxicity among others. Similarly, mTOR inhibitors may cause fatigue, diarrhea, rash, and metabolic as well as electrolyte derangements. Both drugs have significant rates of drug discontinuations and interruptions that can have substantial effects on disease related outcomes. Studies have shown for instance that in patients treated with VEGFR TKIs for $\mathrm{mRCC}$, response rate and PFS, have been correlated to dose intensity [13-15]. One specific study of sunitinib therapy indicated that patients who maintain dose intensity (higher drug exposure) had better survival [14]. As we have learned more of about these agents, it is clear that managing side effects with appropriate follow up, supportive care, and dose modifications as appropriate may be of crucial importance to help maximize efficacy [16]. It should also be noted that there were numerically more nephrectomies done in the ACAD population. It is unclear as to the reason for this, whether related to patient specific (age, comorbidity) or practice specific (inter-disciplinary approach) factors; it is also unclear as to how this finding may have impacted outcomes.

The treatment landscape of mRCC continues to evolve today with the approvals of newer systemic therapies including immunotherapies and combination approaches with often high rates of grade 3 and 4 toxicities [3, 5-7]. Most of these studies have been conducted in high volume academic centers by subspecialists in RCC, resulting in approvals of drug therapies that may not translate easily into community practice. Newer treatments continue to introduce intricacies to selection and sequencing of drugs and managing side effects, which may have noteworthy impacts on patient outcomes.

Further studies determining reasons for disparities in outcomes are essential to our progress. A prospective metastatic renal cell carcinoma (MaRCC) registry has been accrued across sixty academic and community treatment sites to examine real-world treatment patterns and outcomes across the United
States [17]. Data from MaRCC and future prospective observational studies in $\mathrm{mRCC}$ will be important to confirm and understand differences in outcomes between ACAD and COMM sites. By understanding reasons for disparities, we can develop strategies to combat them.

\section{ACKNOWLEDGMENTS}

The authors have no acknowledgments.

\section{FUNDING}

The authors report no funding.

\section{CONFLICT OF INTEREST}

The authors have no conflict of interest to report.

\section{REFERENCES}

[1] Siegel RL, Miller KD, Jemal A. Cancer statistics, 2019. CA Cancer J Clin. 2019;69(1):7-34

[2] Heng DY, Xie W, Regan MM, et al. External validation and comparison with other models of the International Metastatic Renal-Cell Carcinoma Database Consortium prognostic model: A population-based study. Lancet Oncol. 2013;14(2):141-8.

[3] Motzer RJ, Tannir NM, McDermott DF, et al. Nivolumab plus Ipilimumab versus Sunitinib in Advanced Renal-Cell Carcinoma. N Engl J Med. 2018;378(14):1277-90.

[4] Motzer RJ, Escudier B, McDermott DF, et al. Nivolumab versus Everolimus in Advanced Renal-Cell Carcinoma. N Engl J Med. 2015;373(19):1803-13.

[5] McDermott DF, Atkins MB, Motzer RJ, et al. A phase II study of atezolizumab (atezo) with or without bevacizumab (bev) versus sunitinib (sun) in untreated metastatic renal cell carcinoma (mRCC) patients (pts). Journal of Clinical Oncology. 2017;35(6_suppl):431.

[6] Atkins MB, Plimack ER, Puzanov I, et al. Axitinib in combination with pembrolizumab in patients with advanced renal cell cancer: A non-randomised, open-label, dose-finding, and dose-expansion phase 1b trial. Lancet Oncol. 2018.

[7] Choueiri TK, Larkin J, Oya M, et al. Preliminary results for avelumab plus axitinib as first-line therapy in patients with advanced clear-cell renal-cell carcinoma (JAVELIN Renal 100): An open-label, dose-finding and dose-expansion, phase 1b trial. Lancet Oncol. 2018.

[8] Harrison MR, Hirsch BR, George DJ, et al. Real-world outcomes in metastatic renal cell carcinoma: Insights from a Joint Community-Academic Registry. J Oncol Pract. 2014;10(2):e63-72.

[9] Harrison MR, George DJ, Walker MS, et al. "Real world" treatment of metastatic renal cell carcinoma in a joint community-academic cohort: Progression-free survival over three lines of therapy. Clin Genitourin Cancer. 2013;11(4):441-50. 
[10] Motzer RJ, Hutson TE, Tomczak P, et al. Sunitinib versus interferon alfa in metastatic renal-cell carcinoma. N Engl J Med. 2007;356(2):115-24.

[11] Motzer RJ, Hutson TE, Cella D, et al. Pazopanib versus sunitinib in metastatic renal-cell carcinoma. N Engl J Med. 2013;369(8):722-31.

[12] Joshi SS, Handorf EA, Zibelman M, et al. Treatment Facility Volume and Survival in Patients with Metastatic Renal Cell Carcinoma: A Registry-based Analysis. Eur Urol. 2018;74(3):387-93

[13] van der Mijn JC, Mier JW, Broxterman HJ, Verheul HM. Predictive biomarkers in renal cell cancer: Insights in drug resistance mechanisms. Drug resistance updates: Reviews and commentaries in antimicrobial and anticancer chemotherapy. 2014;17(4-6):77-88.

[14] Houk BE, Bello CL, Poland B, et al. Relationship between exposure to sunitinib and efficacy and tolerability endpoints in patients with cancer: Results of a pharmacokinetic/pharmacodynamic meta-analysis. Cancer Chemotherapy and Pharmacology. 2010;66(2):357-71.
[15] Rini BI, Melichar B, Ueda T, et al. Axitinib with or without dose titration for first-line metastatic renal-cell carcinoma: A randomised double-blind phase 2 trial. Lancet Oncol. 2013;14(12):1233-42.

[16] Lainez N, Garcia-Donas J, Esteban E, et al. Impact on clinical practice of the implementation of guidelines for the toxicity management of targeted therapies in kidney cancer. The protect-2 study. BMC Cancer. 2016;16(1):135.

[17] Bhavsar NA, Harrison MR, Hirsch BR, et al. Design and Rationale of the Metastatic Renal Cell Carcinoma (MaRCC) Registry: A Prospective Academic and Community-Based Study of Patients With Metastatic Renal Cell Cancer. Cancer Invest. 2017;35(5):333-44 